\title{
Scalar Mesons and Chiral States
}

\author{
Muneyuki Ishida ${ }^{1}$ and Shin $\operatorname{IsHIDA}^{2 *)}$ \\ ${ }^{1}$ Department of Physics, Meisei University, Tokyo 191-8506. Japan \\ ${ }^{2}$ Research Institute of Quantum Science, College of Science and Technology \\ Nihon University, Tokyo 101-0062, Japan
}

(Received November 29, 2018)

\begin{abstract}
The essential points and physical backgrounds of the covariant level-classification scheme, based on $\tilde{U}(12)_{S F} \otimes O(3,1)_{L}$, are reviewed: This scheme is extended from the nonrelativistic $S U(6)_{S F} \otimes O(3)_{L}$ scheme by introducing the new $S U(2)$-spin ( $\rho$-spin) degree of freedom, which is necessary for covariant description of composite hadrons. Our scheme predicts the existence of new type of chiral mesons and baryons (Chiralons) out of the conventional $S U(6)_{S F} \otimes O(3)_{L}$ scheme. The $\sigma$ nonet is a typical example of chiralons to be assigned to the $(q \bar{q})$ relativistic $S$-wave state. The new narrow mesons $D_{s}(2317) / D_{s}(2463)$ are naturally assigned as the ground-state scalar and axial-vector chiralons in the $(c \bar{s})$ system.
\end{abstract}

\section{$\S 1$. Introduction}

(Difficulty of Non-Relativistic Classification Scheme) The non-relativistic (NR) hadron level-classification scheme with the approximate static-symmetry

$$
S U(6)_{S F} \bigotimes O(3)_{L}
$$

had been successful for these 4 decades, but recently the necessity for covariant classification has been strengthened: Theoretically the QCD, basic dynamics underlying the hadron physics, has the chiral symmetry, "maximally" relativistic symmetry. Phenomenologically the property of $\pi$-meson as a Nambu-Goldstone boson in the case of broken chiral symmetry has been wellknown. Moreover, the existence of light $\sigma$-meson as chiral partner of $\pi$-meson, $\sigma(600)$, which has been a controversial problem for many years, seems now to be confirmed. However, there is no suitable seat prepared for it in the NR scheme.

(Covariant Classification Scheme and Chiral states/Chiralons) Correspondingly to this situation, a few years ago we have proposed ${ }^{1), 2)}$ a covariant levelclassification scheme for hadrons, unifying the seemingly contradictory two, nonrelativistic and extremely relativistic, viewpoints. Here the framework is manifestly Lorentz-covariant and the space for the static symmetry is extended from Eq. (1) to that of

$$
S U(6)_{S F} \bigotimes S U(2)_{\rho} \otimes O(3)_{L}
$$

where a new additional $S U(2)$-space for the $\rho$-spin $(\rho$ - and $\sigma$ - spin being 2 by 2 Pauli-matrices corresponding to the conventional decomposition of 4 by 4 Dirac $\gamma$

\footnotetext{
*) Associate member
} 
matrices: $\gamma \equiv \sigma \otimes \rho)$ is introduced for covariant description of hadron spin-wave function (WF). As a result, in our scheme the squared-mass spectra become globally $\tilde{U}(12)$-symmetric $\left.{ }^{1)}, 3\right)$ and the mass spectra themselves are able to be reconciled with the broken chiral symmetry. The spin WF for meson and baryon systems are given by the Bargmann-Wigner (BW) spinors, ${ }^{2)}$ which are represented, respectively, as the bi-Dirac and tri-Dirac spinors

$$
\begin{aligned}
& \text { mesons } W_{\alpha}^{\beta} \quad=u_{\alpha} \bar{v}^{\beta} \quad: \alpha=\left(\rho_{3}, \sigma_{3}\right), \beta=\left(\bar{\rho}_{3}, \bar{\sigma}_{3}\right) \text {, } \\
& \text { baryons } W_{\alpha_{1} \alpha_{2} \alpha_{3}}=u_{\alpha_{1}} u_{\alpha_{2}} u_{\alpha_{3}}: \alpha_{i}=\left(\rho_{3}^{(i)}, \sigma_{3}^{(i)}\right),
\end{aligned}
$$

where $\left(\alpha, \beta, \alpha_{i}\right)$ denotes the suffices of Dirac spinors of (quark,anti-quark, $i$-th quark) represented by the eigenvalues of $\rho$-spin and $\sigma$-spin.

In light-quark(LL) meson systems the states with the combination of $\left(\rho_{3}, \bar{\rho}_{3}\right)$

$$
(+,+) ;(\text { Pauli }- \text { states }),(+,-),(-,+),(-,-) ;(\text { Chiral states }),
$$

are expected to be realized in nature, while in the heavy-light quark (HL) meson systems only the states with $\left(\rho_{3}, \bar{\rho}_{3}\right)=(+,+)($ :Paulons $)$ and $(+,-)($ :Chiralons $)$ are expetced, reflecting the physical situation that the HL meson system has the nonrelativistic $S U(6)_{S F}$ spin symmetry (the relativistic chiral symmetry) concerning the constituent Heavy quarks (Light quarks). The Pauli-states/Paulons Eq. (4) are also describable in NR scheme, while the chiral states/Chiralons Eq. (4) have appeared first in the covariant scheme and are out of the NR description. Similarly in the light-quark baryon systems, the states with $\left(\rho_{3}^{(1)}, \rho_{3}^{(2)}, \rho_{3}^{(3)}\right)=(+,+,+)($ :Paulons $)$ and $(+,+,-),(+,-,-)($ :Chiralons $)$ are expected to exist.

\section{§2. Description and Level Structure of Hadrons}

(Wave function and Wave Equation) The WF of mesons $\Phi_{A}{ }^{B}$ and baryons $\Phi_{A_{1} A_{2} A_{3}}$ are described systematically as

$$
\begin{array}{cl}
\Phi_{A} B(x, y) \sim \psi_{q, A}(x) \bar{\psi}^{q, B}(y), & \Phi_{A_{1} A_{2} A_{3}} \sim \psi_{q_{1}, A_{1}}\left(x_{1}\right) \psi_{q_{2}, A_{2}}\left(x_{2}\right) \psi_{q_{3}, A_{3}}\left(x_{3}\right), \\
A=(\alpha, a), B=(\beta, b) ; & \alpha, \beta=(1 \sim 4), \quad a, b=(u, d, s, c, b),
\end{array}
$$

and are assumed to satisfy the Klein-Gordon(KG) equation of Yukawa-type ${ }^{4)}$

$$
\left[\left(\partial / \partial X_{\mu}\right)^{2}-\mathcal{M}^{2}\left(r_{\mu}, \partial / \partial r_{\mu} \cdots ; \partial / i \partial X_{\mu}\right)\right] \Phi(X, r \cdots)=0 \text {. }
$$

The operator $\mathcal{M}^{2}$ is assumed to contain no light-quark Dirac matrices $\gamma^{(q)}$ in the ideal limit, leading to the chiral symmetric global structure of squared-mass spectra. The WF is separated into the two parts, the one of plane-wave center of mass motion and the other of internal WF, as

$$
\Phi(X, r \cdots)=\sum_{N} \sum_{\mathbf{P}_{N}, P_{N, 0}>0}\left[e^{i P_{N} \cdot X} \psi_{N}^{(+)}\left(P_{N}, r \cdots\right)+e^{-i P_{N} \cdot X} \psi_{N}^{(-)}\left(P_{N}, r \cdots\right)\right] .
$$

(Expansion of WF on [Spinor $\otimes$ Space-time] eigen-functions) The internal WF with definite total spin $J$ is expanded in terms of respective eigen functions on 
spinor-space and on internal space-time as

$$
\psi_{J, \alpha \cdots}{ }^{\beta \cdots}\left(P_{N}, r \cdots\right)=\sum_{i, j} c_{i j}^{J} W_{\alpha \cdots}^{(i) \beta \cdots}\left(P_{N}\right) O^{(j)}\left(P_{N}, r \cdots\right),
$$

where $W_{\alpha \cdots \cdots}^{(i) \beta \cdots}\left(P_{N}\right)$ and $O^{(j)}\left(P_{N}, r \cdots\right)$ are covariant tensors respectively, in the $\tilde{U}(4)_{D . S}$. (pseudo-unitary Dirac spinor) space and the $O(3,1)_{L}$ (Lorentz-space).

Spin $W F / B W$-spinors As the complete set of spinor-space eigen-functions we choose the BW spinors, defined as solutions of the (local) KG equations

$$
\left[\left(\partial / \partial X_{\mu}\right)^{2}-M^{2}\right] W_{\alpha \cdots}^{\beta \cdots}(X)=0 \stackrel{\text { Moment. Repr. }}{\longrightarrow}\left(P_{\mu}^{2}+M^{2}\right) W_{\alpha \cdots}^{( \pm) \beta \cdots}(P)=0
$$

For the LL-mesons we have the four physical solutions: ${ }^{2)}$

$$
\begin{aligned}
& U_{\alpha}^{\beta}(P) \equiv u_{\alpha}^{(q)}(P) \bar{v}_{(\bar{q})}^{\beta}(P) ; C_{\alpha}^{\beta}(P) \equiv u_{\alpha}^{(q)}(P) \bar{v}_{(\bar{q})}^{\beta}(-P), \\
& D_{\alpha}^{\beta}(P) \equiv u_{\alpha}^{(q)}(-P) \bar{v}_{(\bar{q})}^{\beta}(P) ; V_{\alpha}^{\beta}(P) \equiv u_{\alpha}^{(q)}(-P) \bar{v}_{(\bar{q})}^{\beta}(-P),
\end{aligned}
$$

where $U$ describes the Paulons with $\left(\rho_{3}, \bar{\rho}_{3}\right)=(+,+)$, while $C, D$ and $V$ describe the Chiralons with $\left(\rho_{3}, \bar{\rho}_{3}\right)=(+,-),(-,+)$ and $(-,-)$, respectively. The $U, V$ have $J^{P}=0^{-}, 1^{-}$, while $C, D$ have $J^{P}=0^{+}, 1^{+}$. The eigen-functions of charge conjugation parity are obtained through the superpositions, $W=U \pm V$ and $C \pm D$. Their explicit forms are given by

$$
\begin{array}{rcccccccc}
W_{\alpha}^{\beta}(v): & i \gamma_{5} & i \tilde{\gamma}_{\mu} & -\gamma_{5} v \cdot \gamma & -i \sigma_{\mu \nu} v_{\nu} & 1 & i \gamma_{5} \tilde{\gamma}_{\mu} & -v \cdot \gamma & -\gamma_{5} \sigma_{\mu \nu} v_{\nu} \\
J^{P C}: & 0^{-+} & 1^{--} & 0^{-+} & 1^{--} & 0^{++} & 1^{++} & 0^{+-} & 1^{+}(12) \\
\phi: & P_{s}^{(N)} & V_{\mu}^{(N)} & P_{s}^{(E)} & V_{\mu}^{(E)} & S^{(N)} & A_{\mu}^{(N)} & S^{(E)} & A_{\mu}^{(E)}
\end{array}
$$

The above $W_{\alpha}^{\beta}(v)$ are the tensors of $\tilde{U}(4)\left(\supset S U(2)_{\rho} \otimes S U(2)_{\sigma}\right)$ symmetry, and correspond to all the 16 Dirac $\gamma$-matrices. By including the flavor $\mathrm{SU}(3)$, the LL mesons are classified as $144\left(=12 \times 12^{*}\right)$ representation of $\tilde{U}(12)_{S F}\left(\supset S U(3)_{F} \otimes S U(2)_{\rho} \otimes S U(2)_{\sigma}\right)$.

1),3) In terms of static $S U(6)_{S F}\left(\supset S U(3)_{F} \otimes S U(2)_{\sigma}\right)$, the 144 includes four $36\left(=6 \times 6^{*}\right)$.

As is evidently seen from Eq. (12) and from the chiral transformation on light quarks reducing $\gamma_{5} u(P)=u(-P)$ and $\bar{v}(P) \gamma_{5}=\bar{v}(-P)$, the $\left(P_{s}^{(R)}, S^{R}\right)$ and $\left(V_{\mu}^{(R)}, A_{\mu}^{(R)}\right)$ $(R=N, E)$ form linear representations of chiral symmetry.

For the HL-mesons we have the two physical solutions, $U$ and $C$, given in Eq. (10). They are decomposed into the pseudo-scalars/vectors, and scalars/axialvectors, respectively, as

$$
U_{\alpha}{ }^{\beta} \sim(1-i v \cdot \gamma)\left[i \gamma_{5} P_{s}+i \tilde{\gamma}_{\mu} V_{\mu}\right], \quad C_{\alpha}^{\beta} \sim(1-i v \cdot \gamma)\left[S+i \gamma_{5} \tilde{\gamma}_{\mu} A_{\mu}\right]
$$

The $U$ and $C$ form $\underline{12 *}$ of $\tilde{U}(12)_{S F}$ symmetry. Through the chiral transformation, the former is changed into the latter as $U(P) \gamma_{5}=C(P)$.

The light quark ground state baryons are assigned as $(12 \times 12 \times 12)_{\text {Sym }}=364$ representation of $\tilde{U}(12)_{S F}$ symmetry. The $\underline{364}$ includes the baryons $\left(\underline{\left.182_{B}\right)}\right)$ and antibaryons $\left(182_{\bar{B}}\right)$. In terms of static $S U(6)_{S F}$, the $\underline{182_{B}}$ includes the $\underline{56_{E}}$ (:Paulons) and $\underline{70_{G}}, \overline{56_{F}}$ (:Chiralons) with $\left(\rho_{3}, \rho_{3}, \rho_{3}\right)=(+\overline{,+,+})$ and $(+,+\overline{,-}),(+,-,-)$, 
respectively. Both $\underline{56 E}$ and $\underline{56} 6_{F}$ include $N$-octets and $\Delta$-decouplets with positive parity, while the negative parity $70_{G}$ include $N$-octets with $J=1 / 2$ and $3 / 2, \Delta$ decouplet with $J=1 / 2$ and $\Lambda$-singlet with $J=1 / 2$.

Internal space-time $W F / Y$ ukawa oscillators As the complete set of spacetime eigen-functions we choose the covariant, 4-dimensional Yukawa oscillator functions. By imposing the freezing relative-time condition they become effectively the conventional, 3-dimensional oscillators:

$$
\left\langle P_{\mu} r_{\mu}\right\rangle=\left\langle P_{\mu} p_{\mu}\right\rangle=0 \Rightarrow O(3,1)_{L} \approx O(3)_{L}
$$

(Mass spectra for low-lying mesons and baryons) Since of the static symmetry (2) the global mass spectra are given by

$$
M_{N}^{2}=M_{0}^{2}+N \Omega, N \equiv 2 n+L,
$$

leading to phenomenologically well-known Regge trajectories.

The masses of ground state mesons and baryons are degenerate in the ideal limit, and they split with each others between chiral partners (spin partners) by the bilinear scalar-quark condensates (the perturbative QCD spin-spin interaction).

\section{$\S 3 . \quad$ Candidates for Chiralons/Concluding Remarks}

(Experimental candidates of chiral particles) In our level-classification scheme a series of new type of multiplets, chiralons, are predicted to exist in the ground and the first excited states. Presently we can give only a few experimental candidates or indications for them:

(LL-mesons) One of the most important candidates is the scalar $\sigma$ nonet to be assigned as $S^{(N)}\left({ }^{1} S_{0}\right):\left[\sigma(600), \kappa(900), a_{0}(980), f_{0}(980)\right]$. The existence of $\sigma(600)$ seems to be established ${ }^{5)}$ through the analyses of, especially, $\pi \pi$-production processes. The firm experimental evidences for $\kappa(800-900)$ were reported in the production processes ${ }^{7)} 8$ ). The properties of $\kappa$ are consistent with those given formerly in $K \pi$ scattering phase shift ${ }^{6}$.

In our scheme the two sets of $P_{s^{-}}$and of $V_{\mu}$-nonets are to exist: The vector mesons ${ }^{9)}$ $\rho(1250)$ and $\omega(1250)$, suspected to exist for long time, are naturally able to be assigned as the members of $V_{\mu}^{(E)}\left({ }^{3} S_{1}\right)$-nonet.

Out of the three established $\eta,[\eta(1295), \eta(1420), \eta(1460)]$ at least one extra, plausibly $\eta(1295)$ with the lowest mass, may belong to $P_{s}^{(E)}\left({ }^{1} S_{0}\right)$ nonet.

The two "exotic" particles $\pi_{1}(1400)$ and $\pi_{1}(1600)$ with $J^{P C}=1^{-+}$and $I=1$, observed ${ }^{10)}$ in the $\pi \eta, \rho \pi$ and other channels, may be naturally assigned as the first excited states $S^{(E)}\left({ }^{1} P_{1}\right)$ and $A_{\mu}^{(E)}\left({ }^{3} P_{1}\right)$ of the chiralons.

( HL-mesons) The new narrow mesons $D_{s}(2317) / D_{s}(2463)$, observed recently in the final states $D_{s}^{+} \pi^{0} / D_{s}^{*+} \pi^{0}$, are pointed out ${ }^{11)}$ to be naturally assigned as the ground-state scalar and axial-vector chiralons in the $(c \bar{s})$ system. Their decay properties are well-explained in our scheme. The scalar/axial-vector chiralons are also expected to exist in $c \bar{n}$ system, denoted as $D_{n 0}^{\chi} / D_{n 1}^{\chi}$. Their masses are predicted 
around $2110 / 2250 \mathrm{MeV}$ by using $\mathrm{SU}(3)$ linear $\sigma$ model. Some results in search ${ }^{12)}$ for their existence are reported.

(qqq-baryons) The two facts have been a longstanding problem that the Roper resonance $N(1440)_{1 / 2^{+}}$is too light to be assigned as radial excitation of $N(939)$ and that $\Lambda(1405)_{1 / 2^{-}}$is too light as the $L=1$ excited state of $\Lambda(1116)$. In our new scheme these two problems dissolve ${ }^{13)}$ in principle, because in the ground states there exist the two $\underline{56}$ with positive parity, $\underline{56_{E}} \oplus$ and $\underline{56_{F}} \oplus$, and one negative-parity $\underline{70_{G}}$. The other puzzle, that the predicted width $\Gamma(\overline{\Delta \rightarrow} N \gamma)$ in the conventional treatment is much small compared with the experiment, may also be solved by considering the relativistic effect of the mixing between $56_{E}$ and $56_{F}$. The other problem of extremely small width of $\Delta(1600) \rightarrow N \gamma$ is explained by the orthogonality between WFs of $\Delta(1600)$ and of $\Delta(1232)$ by assuming them to be ground states. ${ }^{13)}$

(Concluding remarks) We have summarized in this talk the essential points of the covariant level-classification scheme, which has, we believe, a possibility to solve the serious problem in hadron spectroscopy mentioned in Introduction. In this connection further investigations, both experimental and theoretical, for chiral states predicted in this scheme, are urgently required for new development of hadron physics.

\section{References}

1) S. Ishida and M. Ishida, Phys. Lett. B539 (2002) 249.

2) S. Ishida, M. Ishida and T. Maeda, Prog. Theor. Phys. 104 (2000), 785.

3) A. Salam, R. Delbourgo and J. Strathdee, Proc. R. Soc. London A284 (1965) 146.

B. Sakita and K. C. Wali, Phys. Rev. B139 (1965) 1335.

4) H. Yukawa, Phys. Rev. 91 (1953), 415, 416.

$5)$ N. A. Törnqvist, summary talk in proceedings of " $\sigma$-Meson 2000", KEK-proceedings $2000-$ 4; NUP-B-2000-1; Soryusiron kenkyu 102 (2001) No.5.

6) S. Ishida et al., Prog. Theor. Phys. 98 (1997), 621.

E. Beveren et al., Z. Phys. C30 (1986), 651.

7) T.Komda in this proceedings. Wu Ning, hep-ex/0304001.

8) C. Gobel, in proc. of Nihon univ. and KEK symp., Ichigaya, Tokyo, Feb 24-26, 2003.

9) M. Oda, in this proceedings.

10) S. U. Chung, summary talk of Hadron99. V. Dorofeev; A. Popov, proc. of Hadron2001.

11) S. Ishida, in this proceedings. M. Ishida and S. Ishida, Prog. Theor. Phys. 106 (2001), 373.

12) I. Yamauchi, in this proceedings.

13) M. Ishida, in proc. of Nihon univ. and KEK symposium, 2003. 